\section{MS3-P14 Tailor-made beams for macromolecular crystals on P14 at PETRAIII}

Anna Polyakova ${ }^{1}$, Gleb Bourenkov ${ }^{1}$, Ivars Karpics ${ }^{1}$, Stefan Fielder $^{1}$, Fang Liu ${ }^{1}$, Thomas R. Schneider ${ }^{1}$

\section{EMBL-Hamburg}

email: anna.polyakova@embl-hamburg.de

The newest addition to the beamline optics of the P14 beamline at PETRAIII for macromolecular crystallography is a transfocator (Vaughan et al, 2011) consisting of compound refractive lenses (CRLs). Acting as an additional focusing device, the CRLs produce beams with increased flux and different focal properties across a wide energy range. For example, the CRLs will enable adjustment of the beam size to match crystals in the size range of $100-500 \mu \mathrm{m}$ with an up to $50 \mathrm{X}$ increase in flux. The transfocator allows the number of CRLs in the beam path to be easily changed, adjusting the focal distance and hence the beam size to match the dimensions of protein crystals of a wide range of sizes. The main objective of this study is to establish the conditions required to adjust the beam to crystals in the size range of $10-500 \mu \mathrm{m}$. Following characterisation of the beam size and flux as obtained by employing different combinations of CRLs, the quality of the diffraction data from several macromolecular crystal systems is evaluated in order to establish optimum conditions. Diffraction data is collected from standard test systems (e.g. lysozyme, insulin, trypsin), as well as more challenging crystals from collaborators from the Karolinska Institute in Sweden. In addition, we aim to make the use of the CRLs available to P14 users through the beamline control software MxCuBE (Gabadinho et al, 2010), enabling rapid and easy adjustment of the beam size. Initial experiments have shown that the CRLs can be successfully used to adjust the size of the beam at P14 in the 20 to 150 micron range for X-ray energies between 6.1-12.7 keV. Diffraction data acquired at various energies have also shown that high quality native and anomalous data can be collected with CRLs in the beam path. These results show promise of the CRLs soon to be made available to P14 users, enabling them to optimise data quality for given crystals by matching the beam properties to the crystal. The project is performed in a collaboration with the groups of Gunter Schneider and Robert Schnell (Karolinska Institute, Stockholm) and is funded by the RAC programme.

Vaughan, G. B. M., et al. (2011). X-ray transfocators: focusing devices based on compound refractive lenses. Journal of Synchrotron Radiation, 18(Pt 2), 125-133.

Gabadinho, J., et al. (2010). MxCuBE: a synchrotron beamline control environment customized for macromolecular crystallography experiments. Journal of Synchrotron Radiation, 17(5), 700-707.

Keywords: X-ray optics, compound refractive lenses, data collection

\section{MS3-P15 P13 and P14, the EMBL Beamlines for Macromolecular Crystallography at PETRA III}

Isabel Bento ${ }^{1}$, Gleb Bourenkov ${ }^{1}$, Michele Cianci ${ }^{1}$, Guillaume Pompidor $^{1}$, Johanna Kallio ${ }^{1}$, Ivars Karpics ${ }^{1}$, Anna Polyakova ${ }^{1}$, Stefan Fiedler ${ }^{1}$, Thomas R. Schneider

1. European Molecular Biology Laboratory, Hamburg Outstation, Notkestrasse 85, 22607 Hamburg, Germany

email: ibento@embl-hamburg.de

EMBL is operating two beamlines for macromolecular crystallography on PETRA III (DESY, Hamburg). Both beamlines are fully tunable and provide a wide range of beam conditions. High flux X-ray beams with adjustable dimensions between 5 and $200 \mu \mathrm{m}$ are available in the energy range between 4 and $18 \mathrm{keV}$. To demonstrate the capability of the beamlines, we will describe and discuss typical experiments including:

- Structure solution via S-SAD phasing using $4 \mathrm{keV}$ X-rays on P13.

- Structure solution using SAD phasing at $6.5 \mathrm{keV}$ on multiple crystals with linear dimensions $<10 \mu \mathrm{m}$.

- Structure solution by molecular replacement from data collected using serial helical scans on micro-crystals presented to the beam at room temperature in CrystalDirect $^{\mathrm{TM}}$ plates.

- Rapid ( $<3$ min) data collection using a CRL-collimated X-ray beam with a 'top-hat' profile.

As a CrystalDirect Harvester system will be installed at EMBL Hamburg in June 2016, we hope to be able to present first results with crystals harvested with this system by the time of the meeting.

Keywords: Beamlines for Macromolecular Crystallography, SAD phasing, Serial Synchrotron Crystallography 